\title{
Pediatric methylation class HGNET-MN1: unresolved issues with terminology and grading
}

\author{
Arnault Tauziède-Espariat ${ }^{1,2 *}$, Mélanie Pagès ${ }^{1,2}$, Alexandre Roux ${ }^{2,3}$, Aurore Siegfried ${ }^{4}$, Emmanuelle Uro-Coste ${ }^{4}$, \\ Yvan Nicaise ${ }^{4}$, Annick Sevely ${ }^{5}$, Marion Gambart ${ }^{6}$, Sergio Boetto ${ }^{7}$, Martin Dupuy ${ }^{8}$, Pomone Richard $^{9}$, \\ Romain Perbet ${ }^{10}$, Matthieu Vinchon ${ }^{11}$, Sabine Caron ${ }^{12}$, Felipe Andreiuolo ${ }^{1,2}$, Albane Gareton ${ }^{1,2}$, \\ Emmanuèle Lechapt ${ }^{1,2}$, Fabrice Chrétien ${ }^{1,2}$, Stéphanie Puget ${ }^{2,13}$, Jacques Grill ${ }^{2,14,15}$, Nathalie Boddaert ${ }^{2,16}$, \\ Pascale Varlet ${ }^{1,2}$ and on behalf of the RENOCLIP-LOC
}

Keywords: MN1, HGNET, Astroblastoma, Meta-analysis

High-grade neuroepithelial tumor with $M N 1$ alteration (HGNET-MN1) is a rare, recently described central nervous system (CNS) entity with a distinct methylation profile, which was formerly part of CNS-primary neuroepithelial tumors (PNET). HGNET-MN1 affects children (77\% of reported cases [1-5]) and are characterized by a recurrent fusion implicating the MN1 (meningioma 1) gene [1]. Limited histopathological and clinical data are available on HGNET-MN1 (44 cases proven by DNA methylation analysis) and a number of outstanding issues still exist [1-5].

Firstly, the histopathological diagnostic criteria are not well established. In Sturm's study, they were initially classified as astroblastomas (16/41, as 4 of our cases) but also as PNETs (12/41), ependymomas (9/41, as 6 of our cases), meningioma (1/41), glioblastoma (1/41), embryonal tumor with multilayered rosettes (1/41), and unclassified tumor (1/41) [1]. These data raise the question of an overlap between a histopathological entity (astroblastoma, considered as a glioma in the current World Health Organization -WHO- classification) and a molecular entity (HGNET-MN1). Secondly, the prognosis of HGNET-MN1 remains unclear. The term "HG" was employed because they were initially described within a cohort of malignant tumors [1]. To explore these issues, we screened our local and the French

\footnotetext{
* Correspondence: a.tauziede-espariat@ghu-paris.fr

${ }^{1}$ Department of Neuropathology, GHU Paris Psychiatrie Neurosciences,

Sainte-Anne Hospital, 1, rue Cabanis, 75014 Paris, France

${ }^{2}$ Paris University France, 75006 Paris, France

Full list of author information is available at the end of the article
}

neuropathological network database ( $n=10$, five of them were briefly mentioned in [6]) and the literature $(n=34)$, for pediatric DNA-methylation proven HGNET-MN1 [1-5]. Here, we describe the molecular, histopathological, clinical and imaging characteristics of pediatric HGNET-MN1.

Including our cases, HGNET-MN1 predominantly concern girls $(90.9 \%, 40 / 44$ cases) and are mainly supratentorial (95.3\%, 41/43 cases) [1,3-5]. Radiologically, all our tumors were well-demarcated from adjacent parenchyma with a multi-nodular aspect. Perilesional edema was not constantly observed. None was calcified and one case was hemorrhagic. They consisted of very large lesions (size ranging from 4.5 to $11.0 \mathrm{~cm}$, mean $7.1 \mathrm{~cm}$ ) with prominent solid portions and multiple necrotic areas but a unique cystic component was not the main tumor feature (Additional file 1: Figure S1 A-D). Thus, the radiological analysis showed that HGNET-MN1 differed from ependymoma, with RELA-fusion, the main pediatric differential diagnosis [6].

In the literature, HGNET-MN1 has been identified in a portion of astroblastomas (39\% in adults compared to $78 \%$ in children) and did not constantly harbour astroblastic features (one case of our series and $8.5 \%$ of reported pediatric cases) [1-5]. In our series, the only constant feature was tumoral fibrous or sclerous stroma (Additional file 1 Figure S1 E-F). They were often macroand microcystic $(n=7)$ (Additional file 1 Figure S1 G-H). They showed a large variety of architectural patterns (Additional file 2 Figure S2 A-E). Cellular atypia was mild 
except in two cases (Additional file 2: Figure S2 F). True ependymal rosettes, lymphocytic infiltrates, eosinophilic granular bodies, and Rosenthal fibers were absent. Mitotic count ranged from 1 to 48 for 10 high-power fields (HPF) and the MIB1 ranged from 2 to $40 \%$ (Fig. 1). Microvascular proliferation and necrosis were observed in 3 and 8 cases, respectively (Fig. 1). The immunohistochemical profile (summarized in Additional file 5 Table S1 and Additional file 3: Figure S3) is also heterogeneous. The literature review and our cases show that $72 \%$ of tumors express glial markers [1-5] and 58\% express neuronal markers [1,5]. Our analysis revealed that $50 \%$ of cases express CK18, and, surprisingly for a primary brain tumor, CD56 was focally or not expressed in 5/10 tumors.

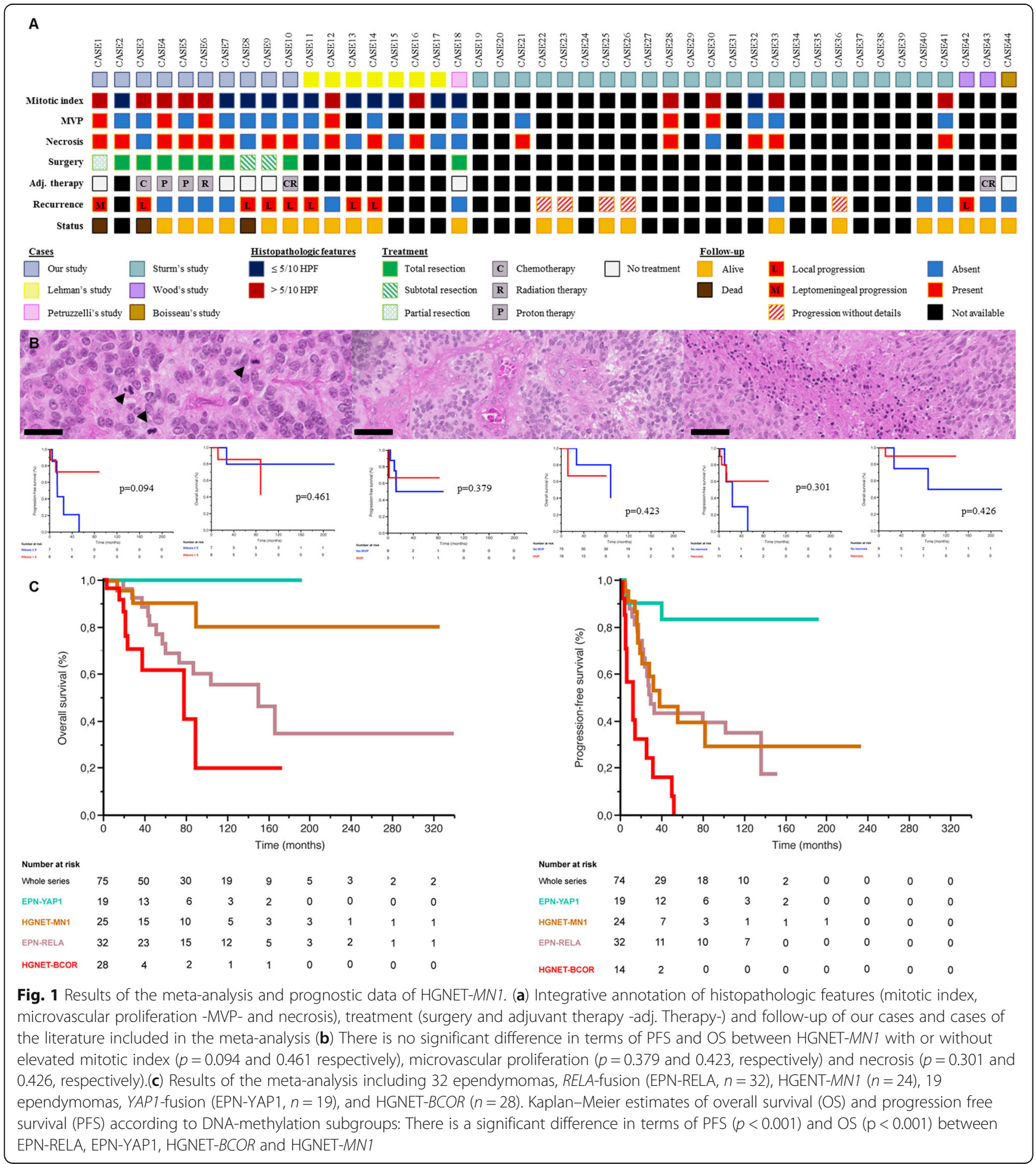


Further fundamental studies and ultrastructural analyses are required to elucidate the cell of origin of HGNETMN1. Thus, HGNET-MN1 present variable morphological and immunophenotypical features.

Our meta-analysis (summarized in Fig. 1, with statistical methodology detailed in Supplementary Data), revealed that during follow-up (mean 81.4 months, range 2.6-324.0), 14/25 patients (56.0\%) experienced tumor progression (8 local, 1 leptomeningeal, and 5 without details) and 3 patients (12.0\%) died (Fig. 1). The mean/ median progression-free survival (PFS) were 43.9/34 months. The mean overall survival (OS) was 81.6 months and the median OS was not reached. Children with HGNET-MN1 present a favourable outcome with $88.0 \%$ survival (median follow-up of 65.5 months), although $48.0 \%$ presented with tumor progression. Three patients (cases 7, 18 and 44) treated only by surgery were alive without tumor progression: one with a long follow-up (229 months) and two with only a short follow-up period (20 and 21 months). Total resection was significantly associated with better OS $(p=0.032)$ and PFS $(p=0.029)$. In univariate analysis, we found significant differences in terms of OS $(p<0.001)$ and PFS $(\mathrm{p}<0.001)$ between HGNET-MN1 and ependymomas with $R E L A$-fusion, ependymomas with $Y A P 1$-fusion and HGNET-BCOR from previous studies [1, 6-9]. Our study has several limitations inherent to its retrospective observational design, but the prognosis does not seem to be as dismal as the term "high-grade" could suggest. No grading criteria have been established for HGNET-MN1. However, the WHO classification mentions histopathologic criteria for malignant astroblastoma [10]: mitotic index $>5 / 10 \mathrm{HPF}$, microvascular proliferation and palisading necrosis. In univariate analysis, these criteria were not significantly, independently or in combination, associated with worse OS $(p=0.461,0.423,0.426$ respectively and in combination $\mathrm{p}=0.461)$ and PFS $(p=0.094,0.379$, 0.301 respectively and in combination $p=0.426$ ) (Fig. 1 , Additional file 4: Figure S4). Moreover, three patients (cases 4, 6 and 12) presenting tumors with these three criteria did not develop progression and are alive (median follow-up of 68.0 months) [5].

In summary, this is the largest series of pediatric HGNET-MN1 with clinical, radiological and histopathological characterization. They present a homogeneous MRI aspect, but a heterogeneous morphology, with inconstant astroblastic pseudorosettes. MN1 fusion must be confirmed to rule out the major differential diagnoses (pleiomorphic xanthoastrocytomas [2-4] and ependymomas [3]). Nevertheless when confronted to our metaanalysis results, naming these tumors has proven difficult. Indeed, both "HGNET" and "astroblastoma" do not completely correlate with grading and morphology. "HGNET" is not a proper designation because the prognosis seems favourable in a subset of cases and further studies are needed to comfort this tendency. "Astroblastoma" is also not quite appropriate since astroblastic pseudorosettes are inconstant and not specific, and a frequent glioneuronal immunophenotype is observed. Our study showed that the two constant histopathological and molecular features of these tumors are the fibrous stroma and the MN1 fusion. Consequently, we would like to suggest the terms "NET, MN1 fusion-positive" or "Fibrous neuroepithelial tumors (FNET), MN1 fusionpositive".

\section{Supplementary information}

Supplementary information accompanies this paper at https://doi.org/10. 1186/s40478-019-0834-z.

\section{Additional file 1: Figure S1. Correlation of radiological and} morphological features in HGNET-MN1. (A) Axial T1 weighted image; (B) Axial T1 post contrast weighted image; (C) Axial T2 weighted image; (D) Coronal T1 post contrast weighted image: they showed a large lesion with multinodular appearance and very important edema. It is a welldemarcated non-intraventricular multinodular tumor with a central solid portion (yellow star) surrounded by multiple cystic components (red asterisks). (E) Multilobular tumor with fibrous central scar delimiting tumo nodules (HPS, 60x). (F) Fibrous scar (yellow star) (HPS, 130x). (G) Macro- and microcystic components (red asterisks) (HPS, 100x). (H) Well-delimitation of the tumor from the brain parenchyma (arrows) (HPS, 200x).

Additional file 2: Figure S2. Variable histopathological patterns of HGNET-MN1. (A) Astroblastic pseudorosettes with variable hyalinization of vessels (HPS, 300x). (B) Ependymoma-like pattern with pseudorosettes (HPS, 400x). (C) Trabecular pattern of the tumor outside of the pseudorosettes (HPS, 400x). (D) Fibrous sclerosis with cordonal structures (HPS, 400x). (E) Myxoid changes with chordoid appearance (HPS, 400X). (F) Tumor cells with nuclear inclusions, atypias and giant cells (HPS, 400x).

Additional file 3: Figure S3. Variable immunohistochemical findings of HGNET-MN1. (A) Diffuse expression of GFAP by glial cells of the pseudorosettes (400x, and insert 400x). (B) Focal expression of GFAP (400x). (C) Diffuse expression of Olig2 in a part of the tumor (400x). (D) Very focal immunoreactivity for Olig2 (400x). (E) Expression of CD56 (400x). (F) Expression of synaptophysin in a part of tumor cells (400x). (G) NeuN immunopositivity (400x). (H) Extra-vascular cellular staining with CD34 in one case (400x). (I) Diffuse expression of cytokeratin 18 (400x).

Additional file 4: Figure S4. Analysis of correlation between malignant criteria defined in astroblastoma applied to HGNET-MN1 with overall survival and progression-free survival. (A) There is no significant difference in terms of PFS between HGNET-MN1 with or without the combination of the three features of anaplasia $(p=0.426)$. (B) There is no significant difference in terms of OS between HGNET-MN1 with or without the combination of the three features of anaplasia $(p=0.461)$.

Additional file 5: Table S1. Immunoprofile of HGNET-MN1 of our series

\begin{abstract}
Acknowledgements
We would like to thank the laboratory technicians at the GHU Paris Neuro Sainte-Anne for their assistance, as well as the Integragen platform for their help with DNA-methylation analyses and the RENOCLIP. The RENOCLIP is the clinico-pathologic network that is instrumental for the central histopathologic review supported by Institut National du Cancer (INCa).
\end{abstract}

\section{Authors' contributions}

ATE, AR, ASe, MG, SB, MD, MV, SC, SP, JG and NB compiled the MRI and clinical records; ATE, MP, ASi, EUC,YN, PR, RP, FA, AG, EL, FC and PV conducted the neuropathological examinations; ATE, MP, EUC, YN and PV conducted the molecular studies; ATE, MP and PV drafted the manuscript; all authors reviewed the manuscript. 


\section{Funding}

The authors declare that they have not received any funding.

\section{Ethics approval and consent to participate}

This study was approved by the GHU Paris Psychiatrie Neurosciences, SainteAnne Hospital's local ethic committee.

\section{Competing interests}

The authors declare that they have no conflicts of interest directly related to the topic of this article.

\section{Author details}

'Department of Neuropathology, GHU Paris Psychiatrie Neurosciences, Sainte-Anne Hospital, 1, rue Cabanis, 75014 Paris, France. ${ }^{2}$ Paris University France, 75006 Paris, France. ${ }^{3}$ Department of Neurosurgery, GHU Paris Psychiatrie Neurosciences, Sainte-Anne Hospital, 75014 Paris, France. ${ }^{4}$ Department of Pathology, Toulouse University Hospital, 31300 Toulouse, France. ${ }^{5}$ Department of Radiology, Purpan University Hospital, 31300 Toulouse, France. ${ }^{6}$ Department of Pediatric Oncology, Toulouse University Hospital, 31300 Toulouse, France. ${ }^{7}$ Department of Neurosurgery, Toulouse University Hospital, 31300 Toulouse, France. ${ }^{8}$ Department of Neurosurgery, Clinique de I'Union, 31240 Saint-Jean, France. 'Laboratory of Pathology, Les Feuillants, 31023 Toulouse, France. ${ }^{10}$ Institute of Pathology, Centre de Biologie Pathologie, Lille University Hospital, 59000 Lille, France. ${ }^{11}$ Neurology, Breast Cancer Department, Oscar Lambret Center, 59000 Lille, France.

${ }^{12}$ Department of Radiology, Centre de Biologie Pathologie, Lille University Hospital, 59000 Lille, France. ${ }^{13}$ Department of Pediatric Neurosurgery, Necker University Hospital, 75015 Paris, France. ${ }^{14}$ CNRS, Gustave Roussy, University Paris-Sud, Université Paris-Saclay, UMR8203 "Vectorologie et Thérapeutiques Anticancéreuses", 94805 Villejuif, France. ${ }^{15}$ Department of Pediatric Oncology, Gustave Roussy, University Paris-Sud, Université Paris-Saclay, 94805 Villejuif, France. ${ }^{16}$ Department of Radiology, Necker University Hospital, 75015 Paris, France.

Received: 7 October 2019 Accepted: 25 October 2019

Published online: 10 November 2019

\section{References}

1. Sturm D, Orr BA, Toprak UH, Hovestadt V, Jones DTW, Capper D et al (2016) New brain tumor entities emerge from molecular classification of CNSPNETs. Cell. 164:1060-1072

2. Boisseau W, Euskirchen P, Mokhtari K, Dehais C, Touat M, Hoang-Xuan K et al (2019) Molecular Profiling Reclassifies Adult Astroblastoma into Known and Clinically Distinct Tumor Entities with Frequent Mitogen-Activated Protein Kinase Pathway Alterations. The Oncologist

3. Lehman NL, Usubalieva A, Lin T, Allen SJ, Tran QT, Mobley BC et al (2019) Genomic analysis demonstrates that histologically-defined astroblastomas are molecularly heterogeneous and that tumors with $\mathrm{MN1}$ rearrangement exhibit the most favorable prognosis. Acta Neuropathol Commun 7:42

4. Wood MD, Tihan T, Perry A, Chacko G, Turner C, Pu C et al (2018) Multimodal molecular analysis of astroblastoma enables reclassification of most cases into more specific molecular entities. Brain Pathol Zurich Switz. 28:192-202

5. Petruzzellis G, Alessi I, Colafati GS, Diomedi-Camassei F, Ciolfi A, Pedace $L$, et al. Role of DNA Methylation Profile in Diagnosing Astroblastoma: A Case Report and Literature Review. Front Genet [Internet]. 2019 [cited 2019 May 24];10. Available from: https://www.ncbi.n/m.nih.gov/pmc/ articles/PMC6502896/

6. Pagès $M$, Pajtler KW, Puget $S$, Castel $D$, Boddaert N, Tauziède-Espariat A et al (2019) Diagnostics of pediatric supratentorial RELA ependymomas: integration of information from histopathology, genetics, DNA methylation and imaging. Brain Pathol Zurich Switz. 29:325-335

7. Andreiuolo F, Varlet $P$, Tauziède-Espariat $A$, Jünger ST, Dörner $E$, Dreschmann $V$ et al (2019) Childhood supratentorial ependymomas with YAP1-MAMLD1 fusion: an entity with characteristic clinical, radiological, cytogenetic and histopathological features. Brain Pathol Zurich Switz. 29: 205-216

8. Ferris SP, Velazquez Vega J, Aboian M, Lee JC, Van Ziffle J, Onodera C et al (2019) High-grade neuroepithelial tumor with BCOR exon 15 internal tandem duplication-a comprehensive clinical, radiographic, pathologic, and genomic analysis. Brain Pathol Zurich Switz
9. Al-Battashi A, Al Hajri Z, Perry A, Al-Kindi H, Al-Ghaithi I (2019) A cerebellar high-grade Neuroepithelial tumor with BCOR alteration in a five-year-old child: a case report. Sultan Qaboos Univ Med J 19:e153-e156

10. Louis DN, Perry A, Reifenberger G, von Deimling A, Figarella-Branger D, Cavenee WK, et al. The 2016 World Health Organization classification of tumors of the central nervous system: a summary. Acta Neuropathol (Berl). 2016;131:803-20. https://doi.org/10.1007/s0040 1-016-1545-1

\section{Publisher's Note}

Springer Nature remains neutral with regard to jurisdictional claims in published maps and institutional affiliations.
Ready to submit your research? Choose BMC and benefit from:

- fast, convenient online submission

- thorough peer review by experienced researchers in your field

- rapid publication on acceptance

- support for research data, including large and complex data types

- gold Open Access which fosters wider collaboration and increased citations

- maximum visibility for your research: over $100 \mathrm{M}$ website views per year

At $\mathrm{BMC}$, research is always in progress.

Learn more biomedcentral.com/submissions 